\title{
Reductive desorption of thiolate from monolayer protected gold clusters
}

\author{
Bernadette M. Quinn and Kyösti Kontturi \\ Laboratory of Physical Chemistry and Electrochemistry, Helsinki University of \\ Technology, P.O. Box 6100, FIN-02015 HUT, Finland
}

\section{Supporting Information}

\section{Preparation of Pt-coat micropipets}

The technique and the manufacture of the coat-pipet assembly have been described in more detail previously. ${ }^{1}$ Briefly, robust micropipets suitable for liquid-liquid electrochemistry were prepared as described by Evans et al. ${ }^{2}$ A coned silver microelectrode was painted with Bright Platinum paint (PBC11611, Johnson Matthey, UK) using a small brush and was fired at $600^{\circ} \mathrm{C}$ for 15 min. The resulting Pt coated tip was then polished with $0.3 \mu \mathrm{m}$ alumina grinding paper (Buehler, US) to expose the silver wire. The silver wire was etched completely with concentrated nitric acid resulting in a Pt coated micropipet with a $25 \mu \mathrm{m}$ inner diameter. The exterior glass was silanized to ensure that the liquid - liquid interface was formed at the $25 \mu \mathrm{m}$ open end of the pipet. This was achieved by immersing the pipet in a $20 \%$ trichloromethylsilane (Sigma) toluene solution for 15 mins while bubbling nitrogen through the end to prevent the silane solution entering the micropipet. The micropipet was then rinsed with toluene and dried in the oven at $100{ }^{\circ} \mathrm{C}$ for 60 mins. Electrical connection was made to the Pt coat. The resulting Pt coated micropipet was filled with the aqueous phase and dipped into the 1,2-dichloroethane (DCE) phase.

A bipotentiostatic arrangement (CHI 900 potentiostat, $\mathrm{CH}$ Instruments, Austin, TX) was used to polarize both the liquid-liquid interface and the Pt outer surface of the pipette. For the polarizable water/DCE interface, a silver wire electrode served as both counter and quasi-reference electrode for the aqueous phase inside the pipette (RE2) and while Ag and Pt wires were used as quasi-reference (RE1) and counter electrodes (CE) in the organic phase. The Pt coat on the external surface of the pipette was the second working electrode (WE1). This is illustrated in the schematic given in Figure 1 (a).

In this manner, the potential applied to the Pt coat/ DCE electrolyte interface $E_{\text {coat }}$ and the interfacial potential difference between the aqueous phase located inside the pipet capillary and the DCE phase, $\Delta_{0}^{\mathrm{w}} \phi$, could be independently controlled. The corresponding electrochemical cells are:

\begin{tabular}{|c|c|c|c|c|c|c|}
\hline Ag & $\begin{array}{c}10 \text { mM BTPPATPBF } 20 \\
\text { (DCE) }\end{array}$ & & $\begin{array}{l}\text { q) } \\
\text { qi }\end{array}$ & $\mathrm{AgCl}$ & Ag & (Cell I) \\
\hline $\mathrm{Ag}$ & $\begin{array}{c}10 \text { mM BTPPATPBF } 20 \\
\text { (DCE) }\end{array}$ & $\mathrm{Pt}$ & (Cell II) & & & \\
\hline
\end{tabular}

where // refers the polarisable water/DCE interface. The preparation of the hydrophobic base electrolyte bis-(triphenylphosphoranylidene) ammonium tetrakis-(pentafluorophenyl)borate (BTPPATPBF 20 ) has been described in detail elsewhere. ${ }^{3}$

The measurements described here involve the localized electrolysis of an electroactive species, (the MPCs) at the Pt coat electrode in the vicinity of a polarized w/DCE interface supported at the tip of a micropipet. Concurrent CVs recorded at the w/DCE interface give an indication of the lipophilicity of the generated charged species or its reactivity. 
To convert the potential applied across the w/DCE interface (cell I) from cell scale to the absolute or Galvani scale $\left(\Delta_{0}^{\mathrm{w}} \phi\right)$, the transfer of a simple ion across the interface (tetraethylammonium, TEA ${ }^{+}$) was used as an internal standard. This involved the addition of a small amount of TEA ${ }^{+}$to the cell $\left(\mathrm{TEATPBF}_{20}\right.$ added to the DCE phase) after the measurement and correlating the measured mid-peak potential on the cell scale with the formal transfer potential on the Galvani scale available in the literature. ${ }^{4}$

The potential applied to the Pt coat electrode (cell II) were corrected to the NHE scale by the addition of ferrocene to the DCE solution after the measurements and correlating its half-wave potential with the formal redox potential given in the literature $\left(E^{0^{\prime}}=0.640 \mathrm{~V}\right.$ vs. $\left.\mathrm{NHE}^{5}\right)$. 


\section{Ion transfer at the micropipet supported liquid-liquid interface}

The micropipet supported liquid-liquid interface has an asymmetric diffusion regime (Figure S1) as mass transfer inside the pipet is controlled by linear diffusion (aqueous phase) and by approximately spherical diffusion outside the pipette (DCE phase). This feature is useful in determining which species is transferring across the interface. ${ }^{1,4}$ For example, as the applied $\Delta_{0}^{\mathrm{w}} \phi$ is made more positive, an anion may transfer from the DCE phase to the aqueous phase or a cation in the opposite direction. At the micropipette interface set up here, the former results in the appearance of a quasi-steady state wave while the latter gives a peak shaped CV response. This is illustrated in Figure S2(A). The steady state diffusion current $\left(I_{\mathrm{ss}}\right)$ can be used as a measure of ion transfer even if the entire voltammogram is not visible within the available $\Delta_{0}^{\mathrm{w}} \phi$ window as illustrated in Fig S2 (B). ${ }^{6}$ This results in an offset current, $I_{\mathrm{ss}}$ throughout the $\Delta_{0}^{\mathrm{w}} \phi$, the height of which is proportional to the diffusion limited transfer of a DCE phase ion to the aqueous phase located inside the pipet (Figure S2 (C)). ${ }^{6}$

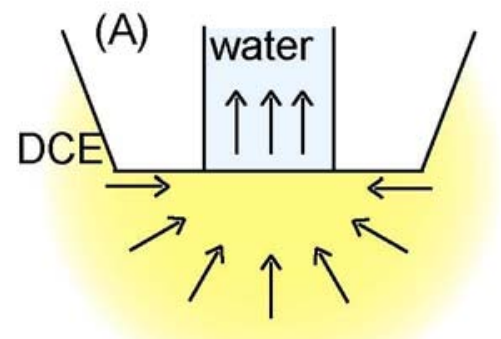

Figure S1 (A) Schematic illustrating the mass transfer regime for ion transfer from the DCE phase outside the pipet to the aqueous phase located inside the pipet. The DCE phase ion is brought to the interface by approximately spherical diffusion and after transferring across the w/DCE interface, is transported away from the interface by linear diffusion in the aqueous phase.

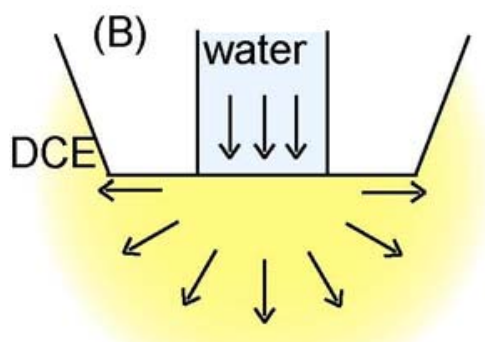

Figure S1 (B) As (A) for an ion transferring from the aqueous phase to the DCE phase. The ion is brought to the interface in this case by linear diffusion and after transfer across the w/DCE interface, is transported away from the interface by approximately spherical diffusion.
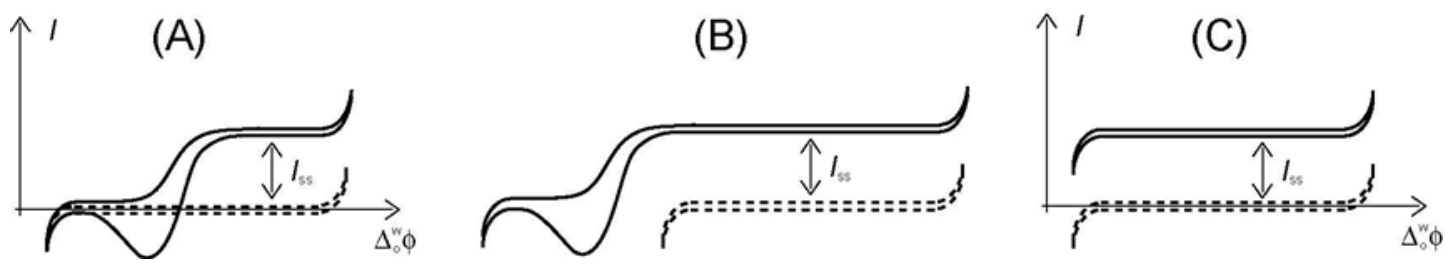

Figure S2 (A) Cartoon depicting the CV response for anion transfer at the w/DCE interface supported at the tip of a micropipet where transfer from DCE to water gives a steady state wave and transfer from water to DCE gives a peak shaped CV. The diffusion limited transfer of the anion from DCE to water is characterized $I_{\mathrm{ss}}$. The available $\Delta_{\mathrm{o}}^{\mathrm{w}} \phi$ window is given by the dashed line and in this case the entire CV for anion transfer (full line) is visible within the $\Delta_{0}^{\mathrm{w}} \phi$ window. For the case where the complete CV for anion transfer is not visible within the available $\Delta_{0}^{\mathrm{w}} \phi$ window (B), the response for the anion transfer overlaps with that for the base electrolyte ions resulting in a composite CV response (C). In this case, there is no zero current region as the anion is transferring at the diffusion limited rate from DCE to water throughout the $\Delta_{0}^{\mathrm{w}} \phi$ window. The shape of the resulting CV is identical to that for the base electrolyte with a current offset equal to $I_{\mathrm{ss}}$. 


\section{Bulk electrolysis of MPCs}

MPCs were oxidized by exhaustive bulk electrolysis in a three-compartment cell as described previously. ${ }^{7}$ Briefly, a DCE solution containing dispersed MPCs and $\mathrm{BTPPATPBF}_{20}$ as base electrolyte were added to the working electrode compartment. The counter electrode compartment was filled with a DCE solution containing only the base electrolyte. A middle sacrificial compartment containing the base electrolyte solution separated the counter and working electrode compartments by dense glass frits on either side. The counter and working electrodes were large area Pt mesh electrodes. A silver wire served as a quasi reference electrode and was dipped in the arm of a luggin capillary added to the working electrode compartment. The luggin capillary contained the same solution as the working electrode compartment. The working electrode solution was stirred by a magnetic stir-bar during electrolysis.

Bulk electrolysis was performed using a PGSTAT 100 (Ecochemie, The Netherlands). Post electrolysis, the MPC solution was cleaned by repetitive precipitation - washing cycles. The equilibrium potential of the MPCs was determined by voltammetry at a Pt electrode (cell II above). The potential at the zero current point was taken as $E_{\text {eq }}$ of the MPC solution.

The counter-ions for the as-prepared $\mathrm{MPC}^{0 /-1}$ (tetraoctylammonium, $\mathrm{TOA}^{+}$from the synthesis conditions employed $\left.{ }^{8}\right)$ and the bulk electrolysed MPCs $\left(\mathrm{TPBF}_{20}{ }^{-}\right.$, the bulk-electrolysis base electrolyte anion) are extremely hydrophobic and do not transfer to the aqueous phase within the $\Delta_{0}^{\mathrm{w}} \phi$ window.
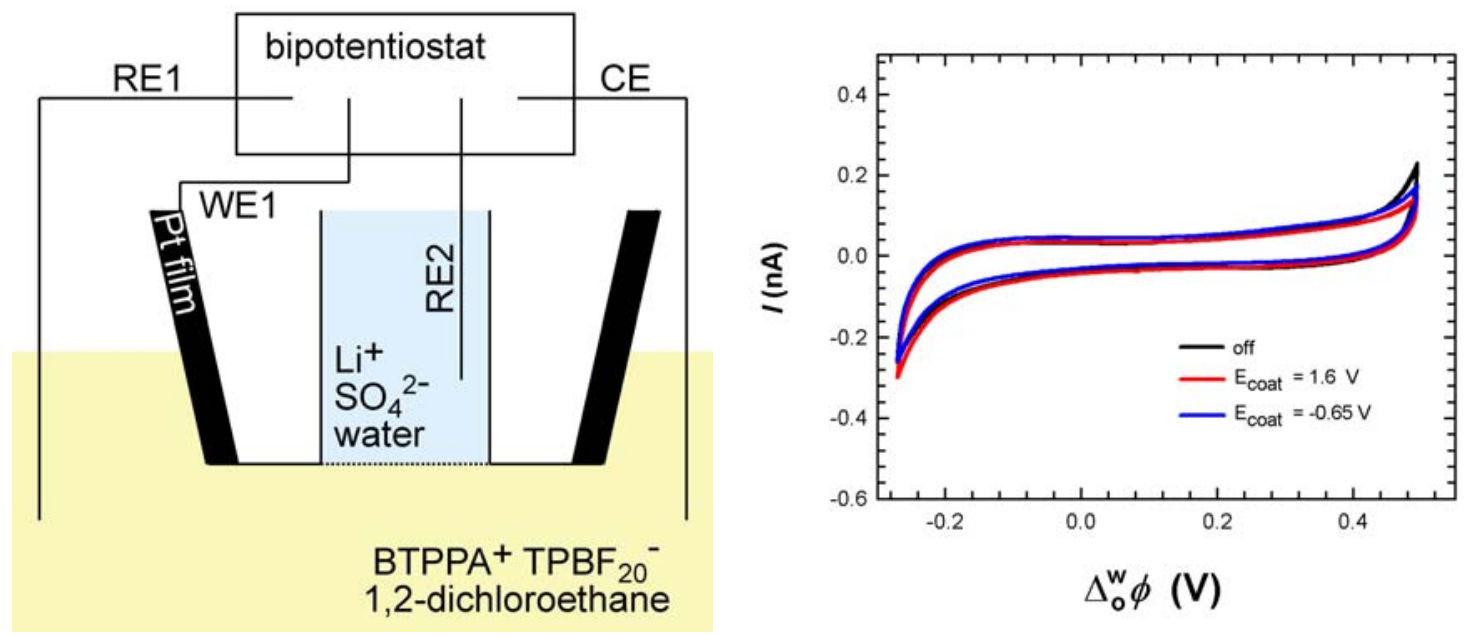

Figure S3. (a) Schematic of the Pt coat - pipet assembly (b) Examples of cyclic voltammograms (CVs) recorded at the pipet supported water / 1,2-dichloroethane (w/DCE) supported interfaces as a function of $\mathrm{Pt}$ electrode bias ( $E_{\text {coat }}$ Vs. NHE). CV scan rate $100 \mathrm{mV} \mathrm{s}^{-1}$. Cells I and II described above were used. In the absence of MPCs added to the DCE phase, biasing the Pt coat electrode at potentials within the Pt/DCE electrolyte electrochemical window (Cell II) does not affect the CV response at the w/DCE interface (Cell I). 


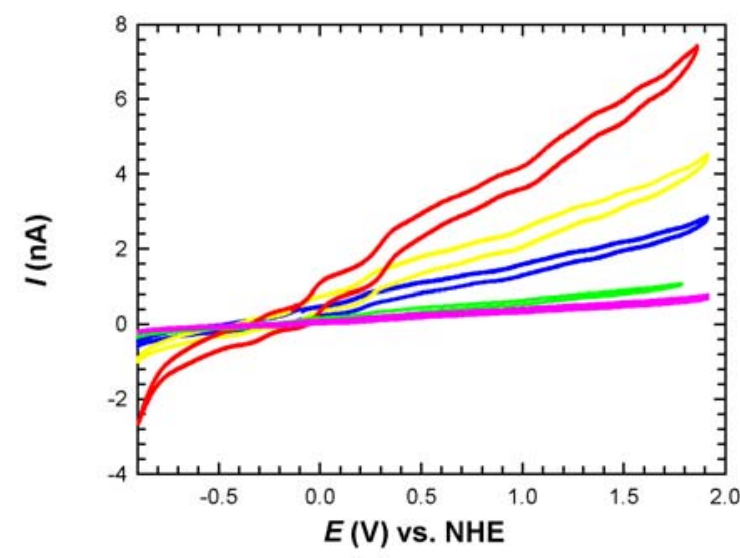

Figure S4. (a) CVs obtained for $\mathrm{MPC}^{0 /-1}$ dispersed in DCE at a Pt microelectrode (diameter $25 \mu \mathrm{m}$ ) at increasing concentrations (95, 140, 380, 650 and 1200 $\mu \mathrm{M})$. (Cell II, scan rate $100 \mathrm{mVs}^{-1}$ ). Cell scale corrected to NHE scale using the oxidation of ferrocene as an internal reference as described above.

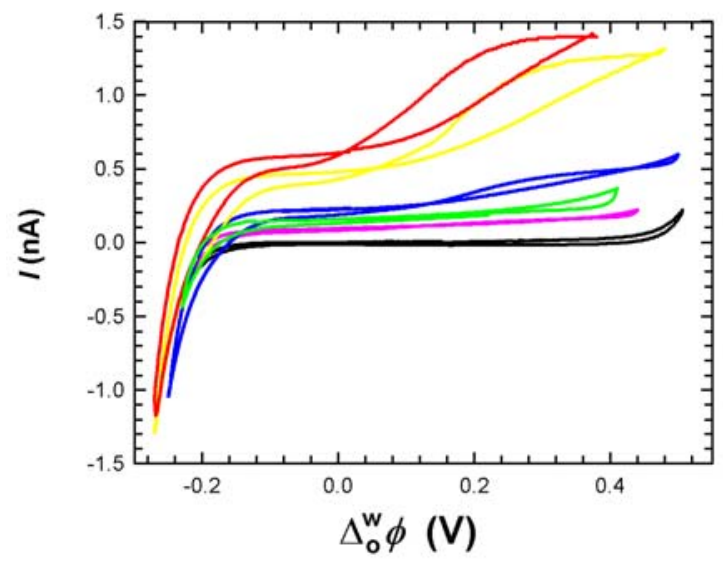

Figure $\boldsymbol{S 4}$ (b) Pipet $\mathrm{CV}$ response at the w/DCE interface for each $\mathrm{MPC}^{0 /-1}$ concentration given in (a). (Cell I, scan rate $25 \mathrm{mVs}^{-1}$ ) The black line shows the background response obtained in the absence of MPCs. For a given MPC preparation, the offset current was proportional to the MPC concentration though there was variation in the absolute current between different preparations. This was ascribed to the concomitant dispersion in $E_{\text {eq }}$ between preparations. At high $\mathrm{MPC}^{0 /-1}$ concentrations, loops are apparent in the $\mathrm{CV}$ response at $\Delta_{\mathrm{o}}^{\mathrm{w}} \phi>0.1 \mathrm{~V}$ due to the surface activity of the thiolate (a short chain surfactant with a polar charged head group and hydrophobic tail). ${ }^{6 a, 9}$ The solubility of alkanethiolates in aquoues solutions is highly dependent on $\mathrm{pH}$. Aggregation in non-alkaline aqueous solution due to low solubility has been widely reported. ${ }^{10}$ 


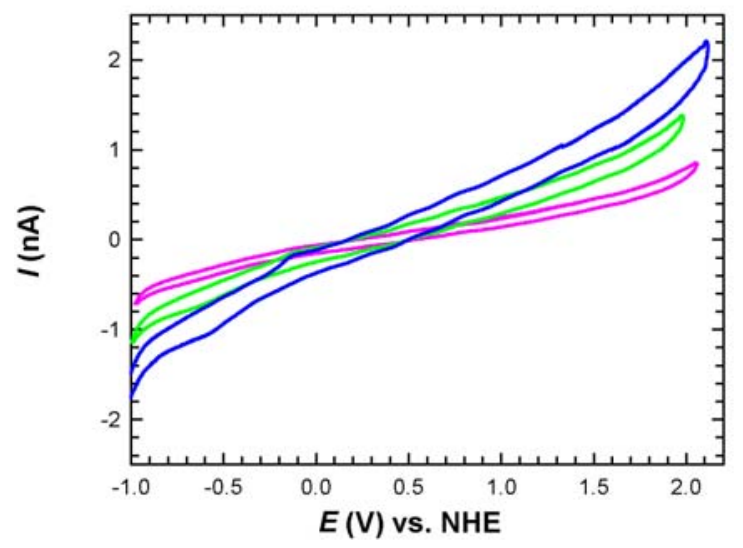

Figure S5. (a) CVs obtained for $\mathrm{MPC}^{2 / 1}$ dispersed in DCE at a Pt microelectrode (Cell II, diameter $25 \mu \mathrm{m}$ ) at increasing concentrations (80, 160 and $245 \mu \mathrm{M}$ ). Experimental details were as in Figure S4 (a).

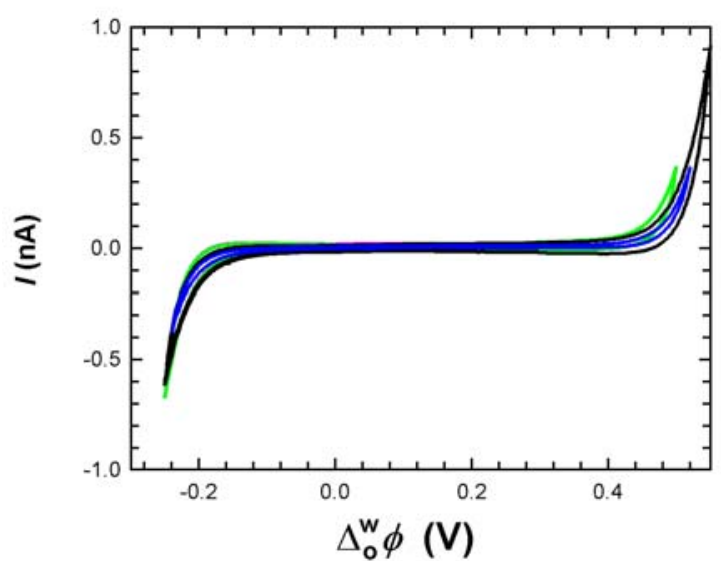

Figure S5 (b) Pipet CV response at the w/DCE interface for each MPC ${ }^{2 / 1}$ concentration given in (a). (Cell I, scan rate $25 \mathrm{mVs}^{-1}$ ) The black line shows the background response obtained in the absence of MPCs. 


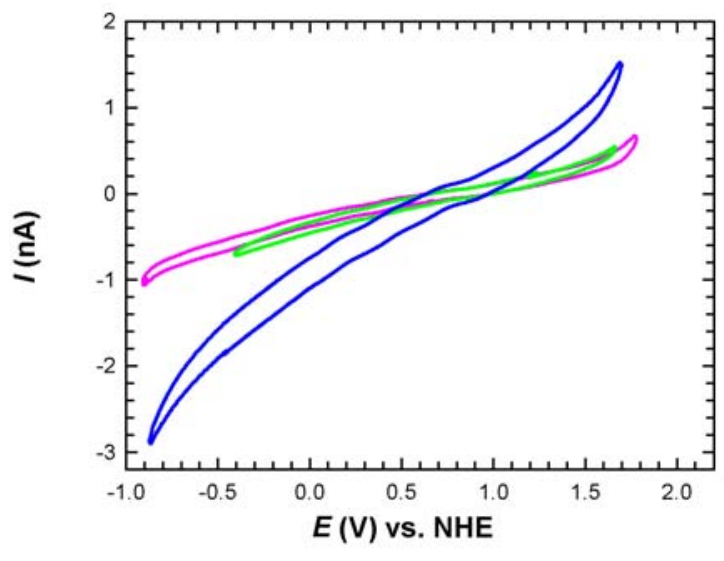

Figure S6. (a) $\mathrm{CVs}$ obtained for $\mathrm{MPC}^{4 / 3}$ dispersed in DCE at a Pt microelectrode (Cell II, diameter $25 \mu \mathrm{m}$ ) at increasing concentrations (105, 40 and $300 \mu \mathrm{M})$. Experimental details were as in Figure S4 (a).

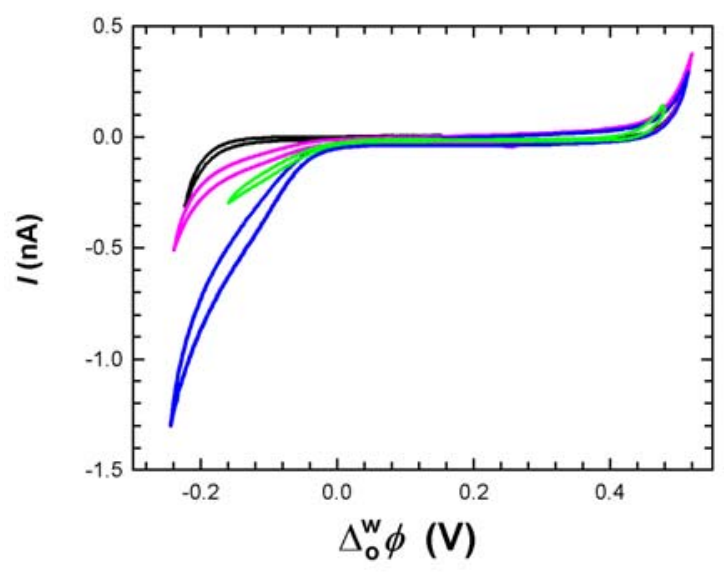

Figure S6 (b) Pipet response at the w/DCE interface for each concentration given in (a). (Cell I, scan rate $25 \mathrm{mVs}^{-1}$ ) The black line shows the background response obtained in the absence of MPCs.

\section{References}

(1) Liljeroth, P.; Quinn, B. M.; Kontturi, K. Electrochem. Commun. 2002, 4, 255. (b) Liljeroth, P.; Quinn, B. M.; Kontturi, K. Langmuir. 2003, 19, 5121.

(2) Evans, N. J.; Gonsalves, M.; Gray, N. J.; Barker, A. L.; Macpherson, J. V.; Unwin, P. R. Electrochem. Commun. 2000, 2, 201.

(3) Fermin, D. J.; Duong, H. D.; Ding, Z.; Brevet, P. F.; Girault, H. H. Phys. Chem. Chem. Phys. 1999, $1,1461$.

(4) Girault, H. H. In Mod. Aspects Electrochem.; White, R. E., Conway, B. E., Bockris, J. O. M., Eds.; Plenum Press: NY, 1993; Vol. 25; p 1.

(5) Fermin, D. J.; Lahtinen, R. In Surfactant Science Series, Volkov, A. G.; Marcel Dekker: New York, 2001; Vol. 95, pp 179-227.

(6) (a) Horrocks, B.R.; Mirkin, M.V. Anal. Chem. 1998, 70, 4653. (b) Hakkarainen, S.; Gilbert, S.L.; Kontturi, A.K.; Kontturi, K. J. Coll. Int. Sci. 2004, 272, 404.

(7) (a) Song, Y.; Huang, T.; Murray, R. W. .J. Am. Chem. Soc. 2003, 125, 11694. (b) Song, Y.: Murray, R. W. .J. Am. Chem. Soc. 2002, 124, 7096. (c) Pietron, J. J.; Hicks, J. F.; Murray, R. W. J. Am. Chem. Soc. 1999, 121, 5565.

(8) Brust, M.; Walker, M.; Bethell, D.; Schiffrin, D. J.; Whyman, R. Chem. Commun. 1994, 801.

(9) (a) Kakiuchi, T.; Chiba, M.; Sezaki,, N.; Nakagawa. M. Electrochem. Comm. 2002, 4, 701. (b) Kasahara T.; Nishi, N.; Yamamoto, M.; Kakiuchi, T. Langmuir, 2004, 20, 875. (c) Kakiuchi, T.; Nishi, N.; Kasahara, T,; Chiba, M. Chem. Phys. Chem. 2003, 4, 179.

(10) Wano, H.; Uosaki, K. Langmuir 2001, 17, 8224. and references therein 